\title{
Alimentation séparée (céréales graines entières + aliment complémentaire granulé) chez les poulets de chair en climat chaud
}

\author{
T. $\mathrm{Yo}^{1}$, M. Picard ${ }^{2}$, H. Guerin ${ }^{3}$, P. Dauvilliers ${ }^{4}$
}

YO (T.), PICARD (M.), GUERIN (H.), DAUVILLIERS (P.). Alimentation séparée (céréales graines entières + aliment complémentaire granulé) chez les poulets de chair en climat chaud. Revue Élev. Méd. vét. Pays trop., 1994, 47 (3) : 319-327

La consommation alimentaire et la croissance des poulets de chair sont limitées par les fortes températures. Une alimentation séparée (AS), composée de céréales "graines entières" et d'un aliment complémentaire, a été comparée avec un aliment complet en farine (ACF) ou en granulés (ACG) pour étudier l'aptitude des poulets à adapter leur ingestion d'énergie et de protéines à leurs besoins. Deux essais ont porté, en saison sèche (SS) et en saison des pluies (SP), plus fraîche, sur 1012 poulets de souche JV15 divisés en 24 lots et sur 6 régimes comprenant un ACF, un ACG et 4 combinaisons d'AS associant du maïs ou du mil à deux formules d'aliments complémentaires. Les poids à 56 jours ont été plus élevés en SP qu'en SS (25 p. 100). Quelle que soit la saison, les croissances en AS ont été de 4 à 7 p. 100 supérieures à celles obtenues en ACF. En revanche, en SS les performances des lots en AS et en ACG étaient globalement identiques (ACG non testé en SP). L'AS a nécessité une semaine d'adaptation après laquelle l'équilibre énergie/protéines fut proche de celui des aliments complets. Elle permet l'utilisation des céréales locales sans frais de transport, de broyage, de mélange ni de granulation. Le prix d'intérêt de l'aliment complémentaire peut être déterminé par ceux des céréales et de l'ACG.

Mots clés : Poulet de chair - Technique et alimentation - Ressource alimentaire - Croissance - Climat tropical - Côte d'lvoire.

\section{INTRODUCTION}

La chaleur constitue l'une des contraintes majeures de l'élevage avicole en zones thopicales. Elle entraîne chez le poulet de chair un ralentissement de la croissance $(1,2,13)$ dû à une importante réduction de l'ingéré alimentaire $(22,33$, 35) et, probablement, à un effet direct sur les mécanismes physiologiques de l'animal $(4,12,14)$.

Plusieurs stratégies nutritionnelles ont été proposées en vue de compenser l'incidence négative de la température en environnement chaud. Elles consistent, généralement, à augmenter la densité nutritionnelle de la ration, notam-

1. IDESSA-DRA, BP 633 Bouaké, Côte d'lvoire.

2. INRA-SRA, 37380 Nouzilly, France.

3. CIRAD-EMVT, 10 rue Pierre Curie, 94704 Maisons-Alfort Cedex, France.

4. UFAC 95450 Vigny, France.

Reçu le 3.5.1994, accepté le 26.10.1994. ment sa teneur en protéines et/ou acides aminés essentiels $(5,29)$ ou à remplacer une partie des glucides de l'aliment par de la matière grasse afin d'abaisser la production d'extra-chaleur chez l'animal $(10,11)$. Cependant, l'utilisation de régimes riches en protéines ne semble pas empêcher une dépression de la croissance du poulet de chair en climat chaud $(16,23,32)$. De même, l'inclusion de matière grasse dans les rations n'a pas toujours donné des résultats concluants $(24,33)$.

II semble donc peu probable d'améliorer de manière significative la productivité des volailles soumises à un stress thermique par le seul remaniement de la composition des aliments composés (14). II apparaît nécessaire de sortir du concept classique d'aliment complet ad libitum au profit de stratégies nouvelles prenant davantage en compte les choix et les rythmes de consommation alimentaire des animaux (25). Dans cette perspective, la méthode d'alimentation séparée, qui consiste à offrir en libre choix différentes fractions d'une ration, pourrait constituer une alternative intéressante. Elle donne aux animaux la possibilité de réguler leur ingéré protéique, indépendamment de l'ingestion d'énergie, et de l'adapter à leur niveau de production ainsi qu'aux conditions climatiques du milieu $(7,21)$.

L'aptitude des volailles de souches sélectionnées à opérer un choix alimentaire correspondant à leurs besoins nutritionnels, en situation de production intensive, a été signalée par quelques auteurs $(8,9,15,26)$. Toutefois, les performances de croissance obtenues chez les poulets de chair avec cette méthode semblent variables. ROSE et KYRIAZAKIS (27), faisant une synthèse de quelques expériences sur l'alimentation séparée (AS) des poulets de chair, ont conclu que l'utilisation de ce système conduit généralement à un gain de poids inférieur d'environ 10 p.100 à celui obtenu avec un aliment complet classique (AC). SCHOLTYSSEK (30) a trouvé une tendance similaire en climat de type tempéré alors que COWAN et MICHIE $(8,9)$ ont montré que la réponse des animaux nourris en AS est fonction de la température. Pour des températures de 16 à $26^{\circ} \mathrm{C}$, ces auteurs ont obtenu un gain de poids identique en $A S$ et en $A C$ alors qu'à $31^{\circ} \mathrm{C}$, l'AS donnait un gain de poids plus élevé que I'AC. SINURAT et BALNAVE (32) et MASTIKA et CUMMING (21) ont également confirmé cet avantage de l'AS à des températures élevées. 
Le régime fractionné, avec des céréales en graincs entières ou broyées, ne semble pas avoir d'effet négatif sur l'indice de consommation (33), le taux de mortalité (30), le rendement carcasse et le taux d'engraissement $(21,31)$. Toutefois, MASTIKA et CUMMING (20) signalent que les souches à croissance rapide, contrairement aux souches lentes, montrent un taux d'engraissement plus élevé en AS qu'en AC.

L'utilisation de céréales graines entières dans un système d'AS offre des perspectives de valorisation directe des surplus de production de céréales, au niveau de la ferme, sans nécessiter un investissement pour le broyage et le mélange des aliments composés. L'AS permet en outre de limiter les coûts' de transport et les contaminations d'aliments broyés au cours du stockage en climat tropical. Cependant, les données actuellement disponibles sur la méthode d'AS ont, en général, été obtenues en climat tempéré ou, plus rarement, en milieu chaud simulé en chambres climatiques. Très peu d'informations existent sur l'utilisation de cette méthode en milieu tropical réel, notamment en Afrique. Cette ćtude vise à tester, en condition tropicale, la réponse des poulets de chair à une alimentation offrant, au choix, une source énergétique sous forme de céréales graines entières et un aliment complémentaire.

\section{MATÉRIEL ET MÉTHODES}

Deux essais ont été conduits au Département des Ressources animales de l'IDESSA (Bouaké, Côte d'Ivoire) en saison sèche et chaude (expérience 1) et en saison pluvieuse et fraîche (expérience 2).

\section{Expérience 1}

Cette étude s'est déroulée du 23 janvier au 19 mars 1992, soit pendant 56 jours. La température au cours de la période expérimentale était, en moyenne, de 25,0 \pm $1,2^{\circ} \mathrm{C}$ pour les minima et $35,5 \pm 1,6^{\circ} \mathrm{C}$ pour les maxima.

\section{Matériel}

L'essai, constitué de six traitements alimentaires, s'est déroulé dans un poulailler au sol, semi-ouvert à ventilation statique, où la litière était composée de balles de riz. Sept cent cinquante-six poussins d'un jour de souche "JV15" pesant en moyenne $41 \mathrm{~g}$ ont été répartis au hasard dans 18 parquets de $6 \mathrm{~m}^{2}$ à raison de 42 animaux par parquet. Chacun des 6 régimes a été distribué à 3 parquets d'animaux. Deux aliments complémentaires protéiques ( $\mathrm{C} 1$ ou $\mathrm{C} 2$ ) ont été utilisés, en les associant au maïs ou au mil (source d'énergie), constituant ainsi 4 combinaisons de céréales et d'aliments protéiques : $\mathrm{C} 1+$ maïs, $\mathrm{C} 1$ + mil, $\mathrm{C} 2$ + maïs, $\mathrm{C} 2$ + mil. Le régime témoin était un aliment complet commercial présenté sous forme de farine (ACF) ou sous forme de granulés (ACG). Les caractéristiques nutritionnelles de ces aliments, déterminées par analyses, sont indiquées dans le tableau I. Les teneurs en énergie métabolisable ont été calculées avec l'équation de JANSSEN (18) pour le maîs et le mil et l'équation CEE indiquée par CARRE et ROZO (6) pour les compléments $\mathrm{C} 1$ et $\mathrm{C} 2$ et les aliments complets. Dans chaque parquet étaient disposées 2 mangeoires: l'une pour la céréale et l'autre pour le complément protéique. Dans les parquets recevant l'ACF ou l'ACG, le même aliment était servi dans les 2 mangeoires.

\section{Méthodes}

L'éclairement total (lumière diurne + lumière artificielle nocturne) était continu pendant les 4 premiers jours puis a été réduit à $18 \mathrm{~h}$ par nycthémère (6-24 h). A partir du $28 \mathrm{e}$ jour, du fait de la forte chaleur diurne, l'éclairement total a été augmenté à $21 \mathrm{~h}(6-3 \mathrm{~h})$ pour le reste de la période d'élevage. Au cours des deux premières semaines, tous les aliments (céréales, aliments complémentaires, aliments complets) étaient distribués sous forme de farine. Le passage aux graines entières de céréales et complémentaires protéiques granulés a été effectué au début de la $3 e$ semaine pour les régimes en AS. L'AC granulé a été servi aux animaux de ce traitement après 3 semaines d'aliment farine.

TABLEAU I Valeurs nutritionnelles des aliments utilisés au cours de l'essai 1.

\begin{tabular}{|c|c|c|c|c|c|c|c|}
\hline & $\mathrm{C} 1$ & $\mathrm{C} 2$ & Maïs & Mil & $\begin{array}{l}\text { Alimentation } \\
\text { de démarrage } \\
(0-21 \mathrm{j})\end{array}$ & $\begin{array}{c}A C F \\
(22-56 j)\end{array}$ & $\begin{array}{c}A C G \\
(22-56 j)\end{array}$ \\
\hline $\begin{array}{l}\text { E.M. Kcal/kg } \\
\text { P.B. (p. 100) } \\
\text { Lysine (p. 100) } \\
\text { A.A.S. (p. 100) } \\
\text { Ca (p. 100) } \\
\text { P. assim. (p. 100) } \\
\text { Mn ppm }\end{array}$ & $\begin{array}{r}2050 \\
40,7 \\
3,2 \\
1,75 \\
3,50 \\
1,70 \\
168\end{array}$ & $\begin{array}{r}2400 \\
39,6 \\
2,7 \\
1,50 \\
3,00 \\
1,50 \\
160\end{array}$ & $\begin{array}{c}3345 \\
8,6 \\
0,23 \\
0,40 \\
0,02 \\
0,05^{\star \star} \\
26\end{array}$ & $\begin{array}{c}3457 \\
8,0 \\
- \\
- \\
0,06 \\
0,08^{* *} \\
20,6\end{array}$ & $\begin{array}{c}3125 \\
21,2 \\
1,10 \\
0,82 \\
1,10 \\
0,50^{*} \\
88\end{array}$ & $\begin{array}{c}3097 \\
19,3 \\
0,96 \\
0,73 \\
0,94 \\
0,50^{\star} \\
100\end{array}$ & $\begin{array}{c}3016 \\
20,8 \\
0,94 \\
0,78 \\
0,99 \\
0,50^{\star} \\
100\end{array}$ \\
\hline
\end{tabular}

* Valeurs communiquées par le fabricant (FACI); ** valeurs extraites de la table INRA (1984). 
A 6,24 et 48 jours d'âge, les animaux ont reçu, dans l'eau de boisson, un traitement vaccinal contre la maladie de Newcastle et à 9 et 26 jours d'âge contre la maladie de Gumboro. De même, tous les animaux ont reçu à titre préventif, toujours dans l'eau de boisson, un traitement anticoccidien à 12,27 et 42 jours d'âge pendant 3 jours consécutifs.

La consommation hebdomadaire de chaque aliment a été mesurée de manière séparée pour le concentré et les céréales. Les animaux ont subi une pesée individuelle à $2,4,6$ et 8 semaines d'âge. La consommation d'eau de chaque parquet a été mesurée à 30 et 45 jours d'âge pendant 2 jours consécutifs. Afin d'établir une relation entre la consommation d'eau, l'ingéré alimentaire et le poids vif, la quantité d'aliment ingérée ainsi que le poids vif ont été calculés par interpolation linéaire à l'aide des 2 moyennes hebdomadaires précédant et suivant les mesures de l'ingéré hydrique.

\section{Expérience 2}

La deuxième expérience s'est déroulée du 9 juillet au 3 septembre 1992. A cette période, la moins chaude de l'année, les températures étaient en moyenne de $22,8 \pm 1,5^{\circ} \mathrm{C}$ pour les minima et de $29,5 \pm 2,2^{\circ} \mathrm{C}$ pour les maxima.

\section{Matériel et méthodes}

L'essai a utilisé 256 poussins chair d'un jour répartis dans 8 parquets de 32 animaux. Tous les animaux ont reçu pendant la période $0-28$ j un aliment complet en farine (ACF). L'expérimentation a porté sur la période 2956 j. Au cours de cette période, l'AS (maïs + complément C1) a été comparée à un $A C$ en farine. La composition des aliments utilisés est indiquée dans le tableau II. Chaque régime a été distribué à 4 lots de 32 poulets de chair entre 29 et 56 jours d'âge. Les mesures relatives au poids vif, à la consommation d'aliment et d'eau ainsi que le programme de prophylaxie étaient identiques à celles de l'expérience 1.

TABLEAU II Caractéristiques des aliments utilisés au cours de l'essai 2.

\begin{tabular}{|l|c|c|c|}
\cline { 2 - 4 } \multicolumn{1}{c|}{} & $\begin{array}{c}\text { Aliment complet } \\
(29-56 \text { jours })\end{array}$ & Maïs & $\begin{array}{c}\text { Complément } \\
\text { C1 }\end{array}$ \\
\hline E.M. (kcal/kg) & 2912 & 3257 & 2050 \\
P.B. (p. 100) & 19,3 & 9,5 & 39,1 \\
Lysine (p. 100) & 1,05 & 0,27 & 3,11 \\
A.A.S. (p. 100) & 0,93 & 0,47 & 1,68 \\
Ca (p. 100) & 1,06 & 0,03 & 3,09 \\
P assim. (p. 100) & $0,5^{\star}$ & $0,05^{\star \star}$ & 1,70 \\
\hline
\end{tabular}

* Valeurs communiquées par le fabricant (FACI) ; ** valeurs extraites de la table INRA (1984).
Ces données ont été soumises à une analyse de variance à l'aide du programme STAT-ITCF et les moyennes des traitements ont été comparées à l'aide du test de comparaison multiple de NEWMAN et KEULS.

\section{RÉSULTATS}

\section{Expérience 1}

\section{Performances de croissance}

L'évolution du poids vif et du gain de poids par période d'élevage sont indiquées dans le tableau III. Dans l'ensemble, l'utilisation du système d'alimentation associant un complément protéique ( $\mathrm{C} 1$ ou $\mathrm{C} 2)$ à une céréale (maïs ou mil) a permis d'obtenir une croissance pondérale régulière. II n'y a pas eu de différence significative entre les poids vifs à 8 semaines obtenus avec les 4 régimes en AS $(p>0,05)$. L'AS a permis un démarrage significativement plus rapide par rapport à la ration commerciale témoin : les poids à 2,4 et 6 semaines (mis à part le régime $\mathrm{C} 1+$ mil à 6 semaines) en $A S$ sont plus élevés qu'avec l'AC $(p<0,01)$. Cependant, au cours des deux dernières périodes, les animaux recevant l'AC granulé ont pu rattraper leur retard de croissance. L'AC granulé donne à 8 semaines un poids vif plus élevé que l'AC en farine $(p<0,05)$ et comparables à ceux obtenus avec les régimes AS ( $p>0,05)$ (tabl. III).

\section{Ingestion et efficacité de transformation de l'aliment}

L'ingestion d'aliment, d'énergie, de protéines brutes et les indices de consommation sont indiqués dans le tableau IV. La quantité totale d'aliment ingéré par jour augmente régulièrement, passant de $18 \mathrm{~g} / \mathrm{j}$ au cours de la première semaine à environ $130 \mathrm{~g} / \mathrm{j}$ au cours de la $7 \mathrm{e}$ semaine d'âge. Au cours de la dernière semaine, on observe une relative diminution de l'ingestion sur les régimes $A S$. Cette diminution pourrait être due, en partie, aux difficultés de conservation du complément protéique.

Pour les rations $\mathrm{C} 1+$ maïs, $\mathrm{C} 1+$ mil et $\mathrm{C} 2+$ mil, on a observé que la part du complément dans la ration quotidienne, de 50 p.100 au cours de la première semaine, a été réduite à $30-36$ p. 100 au cours des 5 semaines suivantes et à $26-29$ p.100 au cours des 7 e et 8 e semaines. Toutefois, pour les régimes séparés avec du maïs, la part de compléments dans les rations ingérées était relativement élevée au cours de la troisième semaine (41 p.100 pour le $\mathrm{C} 1$ et $45 \mathrm{p} .100$ pour le $\mathrm{C} 2$ ), en raison de la présence de quelques impuretés dans le maïs. Le tamisage des grains de maïs a permis de résoudre ce problème pour le régime $\mathrm{C} 1$ + maïs. En revanche, pour le régime $\mathrm{C} 2$ + maîs, la part du complément dans la ration ingérée est restée élevée au cours des 5 premières semaines (42 à 50 p. 100). Ce taux ne s'est abaissé à un niveau comparable aux autres qu'au cours des 3 dernières semaines ( 28 à 33 p. 100). Sur les 8 semaines d'élevage, le taux moyen 
T. Yo M. Picard H. Guerin P. Dauvilliers

TABLEAU III Evolution du poids vif et du gain moyen quotidien (GMQ) (g/poulet/j) en fonction de l'âge.

\begin{tabular}{|c|c|c|c|c|c|c|}
\hline Périodes & $\mathrm{C} 1$ + maïs & $\mathrm{C} 1+\mathrm{mil}$ & $\mathrm{C} 2$ + maïs & $\mathrm{C} 2+\mathrm{mil}$ & $A C F$ & $A C G$ \\
\hline $\begin{array}{l}\text { Poids vif }(\mathbf{g}) \\
1 \text { jour } \\
14 \text { jours } \\
28 \text { jours } \\
42 \text { jours } \\
56 \text { jours }\end{array}$ & $\begin{array}{c}41 a \\
339 \pm 27 a \\
952 \pm 99 a \\
1644 \pm 170 a \\
2107 \pm 248 a\end{array}$ & $\begin{array}{c}41 a \\
314 \pm 28 a \\
926 \pm 111 a \\
1557 \pm 206 b c \\
2061 \pm 240 a b\end{array}$ & $\begin{array}{c}41 a \\
334 \pm 29 a \\
978 \pm 98 a \\
1655 \pm 159 a \\
2137 \pm 259 a\end{array}$ & $\begin{array}{c}41 a \\
313 \pm 28 a \\
936 \pm 119 a \\
1605 \pm 189 a b \\
2099 \pm 275 a\end{array}$ & $\begin{array}{c}41 a \\
227 \pm 42 b \\
759 \pm 117 b \\
1424 \pm 186 d \\
2022 \pm 257 b\end{array}$ & $\begin{array}{c}41 a \\
235 \pm 36 b \\
796 \pm 96 b \\
1521 \pm 148 c \\
2103 \pm 244 a\end{array}$ \\
\hline $\begin{array}{l}\text { GMQ (g/an/jour) } \\
0-2 \text { semaines } \\
\text { 3-4 semaines } \\
5-6 \text { scmaines } \\
7-8 \text { semaines }\end{array}$ & $\begin{array}{c}21,2 a \\
43,9 a b \\
49,4 a \\
33,1 b\end{array}$ & $\begin{array}{c}19,5 a \\
43,7 a b \\
45,1 a \\
36,0 b\end{array}$ & $\begin{array}{l}20,9 a \\
46,0 a \\
48,4 a \\
34,4 b\end{array}$ & $\begin{array}{c}19,4 \mathrm{a} \\
44,5 \mathrm{ab} \\
47,8 \mathrm{a} \\
35,3 \mathrm{~b}\end{array}$ & $\begin{array}{l}13,3 \mathrm{~b} \\
30,0 \mathrm{c} \\
47,5 \mathrm{a} \\
42,7 \mathrm{a}\end{array}$ & $\begin{array}{c}13,9 \mathrm{~b} \\
40,0 \mathrm{bc} \\
51,8 \mathrm{a} \\
41,6 \mathrm{a}\end{array}$ \\
\hline
\end{tabular}

Les moyennes figurant sur la même ligne et portant la même lettre ne sont pas significativement différentes $(p>0,05)$.

TABLEAU IV Consommation quotidienne moyenne d'aliment, d'énergie et de protéines et indice de consommation pour la période (0-8 S).

\begin{tabular}{|c|c|c|c|c|c|c|}
\hline & $\mathrm{C} 1$ + maïs & $\mathrm{C} 1+\mathrm{mil}$ & $\mathrm{C} 2$ + maïs & $\mathrm{C} 2+\mathrm{mil}$ & Aliment farine & Aliment granulé \\
\hline $\begin{array}{l}\text { Consommation/jour } \\
\text { Céréales (g/an/j) } \\
\text { Complément }(\mathrm{g} / \mathrm{an} / \mathrm{j}) \\
\text { Total (g/an/j) } \\
\text { Pourcentage de complément } \\
\text { Protéines }(\mathrm{g} / \mathrm{an} / \mathrm{j}) \\
\text { Energie }(\mathrm{kcal} / \mathrm{an} / \mathrm{j})\end{array}$ & $\begin{array}{c}61 \mathrm{a} \\
30 \mathrm{~b} \\
91 \mathrm{a} \\
33 \mathrm{p} .100 \\
17,5 \mathrm{a} \\
266 \mathrm{bc}\end{array}$ & $\begin{array}{c}61 a \\
29 b \\
90 a \\
32,2 p .100 \\
16,7 b \\
270 b\end{array}$ & $\begin{array}{c}58 a \\
33 a \\
91 a \\
36,3 \text { p. } 100 \\
18 a \\
274 b\end{array}$ & $\begin{array}{c}61 \mathrm{a} \\
29 \mathrm{~b} \\
90 \mathrm{a} \\
32,1 \mathrm{p} .100 \\
16,5 \mathrm{~b} \\
282 \mathrm{a}\end{array}$ & $\begin{array}{c}- \\
- \\
83 \mathrm{~b} \\
16,3 \mathrm{~b} \\
258 \mathrm{c}\end{array}$ & $\begin{array}{c}- \\
- \\
87 a b \\
1 \\
18,1 a \\
264 b c\end{array}$ \\
\hline $\begin{array}{l}\text { Indice de consommation } \\
\text { IC (g aliment/g gain) } \\
\text { Energie (kcal/g gain) } \\
\text { Protéines (g PB/gain) }\end{array}$ & $\begin{array}{c}2,49 a \\
7,20 a \\
0,48 a b\end{array}$ & $\begin{array}{c}2,50 a \\
7,49 a \\
0,46 a b\end{array}$ & $\begin{array}{c}2,43 a \\
7,31 a \\
0,48 a b\end{array}$ & $\begin{array}{l}2,46 a \\
7,68 a \\
0,45 b\end{array}$ & $\begin{array}{c}2,35 a \\
7,29 a \\
0,46 a b\end{array}$ & $\begin{array}{l}2,36 a \\
7,16 a \\
0,49 a\end{array}$ \\
\hline
\end{tabular}

Les moyennes figurant sur la même ligne et portant la même lettre ne sont pas significativement différentes $(p>0,05)$.

TABLEAU V Consommation d'eau (en ml/poulet/jour).

\begin{tabular}{|c|c|c|c|c|c|c|c|}
\hline \multicolumn{2}{|c|}{$\begin{array}{l}\text { Âge } \\
\text { (Température) }\end{array}$} & \multirow{2}{*}{$\begin{array}{c}\text { C1 + maïs } \\
304 a \\
2,69 a \\
29\end{array}$} & \multirow{2}{*}{$\begin{array}{c}\mathrm{C} 1+\mathrm{mil} \\
242 \mathrm{~b} \\
2,23 \mathrm{bc} \\
24\end{array}$} & \multirow{2}{*}{$\begin{array}{c}\mathrm{C} 2+\text { maïs } \\
299 \mathrm{a} \\
2,77 \mathrm{a} \\
28\end{array}$} & \multirow{2}{*}{$\begin{array}{c}\mathrm{C} 2+\mathrm{mil} \\
258 \mathrm{~b} \\
2,43 a b \\
25\end{array}$} & \multirow{2}{*}{$\begin{array}{c}\text { ACF } \\
186 \mathrm{c} \\
2,09 \mathrm{c} \\
22\end{array}$} & \multirow{2}{*}{$\begin{array}{c}A C G \\
239 b \\
2,58 a \\
27\end{array}$} \\
\hline $\begin{array}{l}30 \text { jours } \\
\left(32^{\circ} \mathrm{C}\right)\end{array}$ & $\begin{array}{l}\text { Eau }(\mathrm{ml} / \mathrm{an} / \mathrm{j}) \\
\text { Eau/aliment }(\mathrm{ml} / \mathrm{g}) \\
\text { Eau/poids vif }(\mathrm{p} .100)\end{array}$ & & & & & & \\
\hline $\begin{array}{l}45 \text { jours } \\
\left(30^{\circ} \mathrm{C}\right)\end{array}$ & $\begin{array}{l}\text { Eau }(\mathrm{ml} / \mathrm{an} / \mathrm{j}) \\
\text { Eau/aliment }(\mathrm{ml} / \mathrm{g}) \\
\text { Eau/poids) vif }(\mathrm{p} .100)\end{array}$ & $\begin{array}{c}318 \mathrm{a} \\
2,46 \mathrm{ab} \\
18\end{array}$ & $\begin{array}{c}281 \mathrm{~b} \\
2,20 \mathrm{~b} \\
17\end{array}$ & $\begin{array}{c}340 \mathrm{a} \\
2,61 \mathrm{a} \\
19\end{array}$ & $\begin{array}{l}313 a b \\
2,41 a b \\
18\end{array}$ & $\begin{array}{c}316 a b \\
2,44 a b \\
20\end{array}$ & $\begin{array}{l}354 a \\
2,71 a \\
22\end{array}$ \\
\hline
\end{tabular}


de complément protéique a été de $33,32,2$ et 32,1 p.100 respectivement pour les régimes $C 1+$ maïs, $C 1+$ mil et $\mathrm{C} 2$ + mil contre 36,3 p. 100 pour le régime $\mathrm{C} 2$ + maïs.

Pendant la période de 0-6 semaines, les animaux en AS ont ingéré plus d'énergie que ceux recevant un $A C$, présenté en farine ou en granulé. Les animaux en $A C$ ont cependant quelque peu compensé leur retard par une ingestion énergétique plus élevée au cours de la période 7-8 semaines. Sur la période totale, l'ingéré énergétique le plus élevé est apparu avec le traitement $\mathrm{C} 2$ + mil et le plus faible avec le traitement $A C$ en farine. Les traitements $C 1+$ maïs, $\mathrm{C} 1$ + mil, $\mathrm{C} 2$ + maïs et l'aliment granulé ne diffèrent pas statistiquement $(p>0,05)$ (tabl. IV).

Sur l'ensemble de la période d'élevage, 0-8 semaines, les rations $A S$ à base de maïs et l'AC en granulé ont permis une ingestion de protéines plus élevée que l'aliment farine et les rations AS à base de mil. L'évolution des ratios énergie/protéines des rations sélectionnées par les animaux est représentée dans la figure 1 pour les périodes $0-4$ et $5-8$ semaines. Cette figure montre que le rapport énergie/protéines des rations sélectionnées par les animaux tend à augmenter avec l'âge. Cependant, son évolution est irrégulière entre les $3 e$ et $5 e$ semaines. L'indice de consommation ( $g$ aliment/g de gain) et l'indice de consommation énergétique (Kcal/g gain) sont identiques pour tous les régimes $(p>0,05)$. Seul l'indice de consommation protéique ( $g$ protéines/g gain) du régime $\mathrm{C} 2+$ mil est significativement inférieur à celui de l'ACG $(p<0,05)$.

\section{Consommation d'eau}

Les analyses de variance montrent que l'ingéré hydrique du poulet est non seulement fonction de la quantité d'aliment consommée mais aussi de la composition de la ration ainsi que de la forme sous laquelle celle-ci est présentée. Une première analyse de variance, limitée aux 4 régimes d'AS, et prenant en compte le type de céréales (facteur 1) et le type de complément protéique (facteur 2) montre à 30 j d'âge que la présence du maîs dans la ration entraîne une augmentation de la consommation d'eau, indépendamment de la quantité d'aliment ingérée $(p<0,001)$. A 45 jours d'âge, on observe que la présence du maîs ou du $\mathrm{C} 2$ dans une ration a un effet significatif sur la quantité d'eau ingérée ainsi que sur le rapport eau/aliment $(p<0,05)$.

Une deuxième analyse, unifactorielle, prenant en compte le facteur mode d'alimentation (les 6 traitements) a permis de comparer les moyennes (tabl. V). On remarque notamment une consommation d'eau et un rapport eau/aliment plus élevés pour l'aliment complet en granulé que pour celui en farine. La différence à 45 j n'est cependant pas significative $(p>0,05)$.

\section{Expérience 2}

\section{Performances de croissance}

Le poids vif des poulets de 4 à 8 semaines est indiqué dans le tableau VI. L'évolution du poids vif montre une nette supériorité de l'AS sur l'AC présenté en farine $(p<0,05)$. La différence est déjà de $100 \mathrm{~g}$ après seulement 2 semaines de régime; ce qui dénote la facilité d'adaptation des poulets de chair à ce mode d'alimentation. En fin d'essai à 8 semaines, on observe une différence de poids de $170 \mathrm{~g}$ au profit du régime expérimental.

\section{Ingestion et indices de consommation}

La consommation moyenne d'aliment (29-56 j) est plus élevée pour les animaux recevant l'AS $(p<0,05)$. Le taux de complément dans la ration est de 46 p. 100 au cours de la première semaine de régime (semaine 5). Ce taux descend ensuite à 38,29 puis 27 p. 100 respectivement pour les semaines 6,7 et 8 .

Les ingestions d'aliments, d'énergie et de protéines des poulets recevant une AS sont plus élevées $(p<0,05)$. Toutefois, les indices de consommation pour les périodes 5-6 et 7-8 semaines sont identiques pour les deux modes d'alimentation (tabl. VII).

TABLEAU VI Évolution du poids et du gain de poids vif.

\begin{tabular}{|c|c|c|}
\hline & $\begin{array}{l}\text { Aliment complet } \\
\text { farine }\end{array}$ & $\begin{array}{l}\text { Aliment séparé } \\
\text { (maïs + C1) }\end{array}$ \\
\hline $\begin{array}{l}\text { Poids vif (g/an) } \\
4 \text { semaines } \\
6 \text { semaines } \\
8 \text { semaines }\end{array}$ & $\begin{array}{c}731 a \\
1592 \pm 175 b \\
2512 \pm 261 b\end{array}$ & $\begin{array}{c}743 a \\
1698 \pm 180 a \\
2680 \pm 347 a\end{array}$ \\
\hline $\begin{array}{l}\mathrm{GMQ}^{\star}(\mathrm{g} / \mathrm{an} / \mathrm{j}) \\
5-6 \text { semaines } \\
7-8 \text { semaines }\end{array}$ & $\begin{array}{l}61,5 b \\
65,7 a\end{array}$ & $\begin{array}{l}68,2 a \\
70,1 a\end{array}$ \\
\hline
\end{tabular}

+ GMQ : gain moyen quotidien.

Les moyennes tigurant sur la mème ligne et portant la mëme lettre ne sont pas significativement différentes $(p>0.05)$.

TABLEAU VII Consommation quotidienne moyenne d'aliment, d'énergie et de protéines et indice de consommation pour la période : $29-56$ jours.

\begin{tabular}{|l|c|c|}
\hline & $\begin{array}{c}\text { Aliment } \\
\text { complet } \\
\text { en farine }\end{array}$ & $\begin{array}{c}\text { Aliment } \\
\text { séparé } \\
\text { maïs + C1 }\end{array}$ \\
\hline Consommation & - & 111 \\
Céréales (g/poulet/jour) & - & 57 \\
Cornplément (g/poulet/jour) & $154 \mathrm{a}$ & $168 \mathrm{~b}$ \\
$\begin{array}{l}\text { Total (g/poulet/jour) } \\
\text { Pourcentage de complément }\end{array}$ & - & $33,9 \mathrm{p} .100$ \\
Protéines (g/poulet/jour) & $29,7 \mathrm{a}$ & $33,7 \mathrm{~b}$ \\
Energie (kcal/poulet/jour) & $448 \mathrm{a}$ & $478 \mathrm{~b}$ \\
\hline Indice de consommation & & \\
IC (g aliment/g gain) & $2,42 \mathrm{a}$ & $2,42 \mathrm{a}$ \\
Energie (kcal/g gain) & $7,04 \mathrm{a}$ & $6,87 \mathrm{a}$ \\
Protéines (g PB/g gain) & $0,47 \mathrm{a}$ & $0,49 \mathrm{a}$ \\
\hline
\end{tabular}

Les moyennes figurant sur la même ligne et portant la même lettre ne sont pas significativement différentes $(p>0,05)$. 


\section{Consommation d'eau}

A 33 jours, la quantité d'eau consommée des poulets varie significativement avec le mode de distribution de l'aliment et est augmentée avec l'aliment séparé maïs + C1. A 47 jours, la différence entre traitements n'est plus significative (tabl. VIII). Lorsque l'on ramène l'ingéré hydrique à la consommation d'aliment ou au poids vif des animaux, il n'apparaît pas de différence significative entre les 2 régimes à 33 ou 47 jours d'âge $(p>0,05)$.

TABLEAU VIII Consommation hydrique des animaux (en grammes).

\begin{tabular}{|c|c|c|c|}
\hline \multicolumn{2}{|c|}{$\begin{array}{l}\text { Âge } \\
\text { (Température) }\end{array}$} & \multirow{2}{*}{$\begin{array}{c}\begin{array}{c}\text { Aliment } \\
\text { complet } \\
\text { en farine }\end{array} \\
224 \mathrm{~b} \\
\begin{array}{c}1,97 \mathrm{a} \\
24\end{array}\end{array}$} & \multirow{2}{*}{$\begin{array}{c}\begin{array}{c}\text { Aliment } \\
\text { séparé }\end{array} \\
\text { maïs + C1 } \\
279 a \\
2,28 a \\
28\end{array}$} \\
\hline $\begin{array}{l}33 \text { jours } \\
\left(24^{\circ} \mathrm{C}\right)\end{array}$ & $\begin{array}{l}\text { Eau }(\mathrm{ml} / \mathrm{an} / \mathrm{j}) \\
\text { Eau/aliment }(\mathrm{ml} / \mathrm{g}) \\
\text { Eau/poids vif }(\mathrm{p} .100)\end{array}$ & & \\
\hline $\begin{array}{l}47 \text { jours } \\
\left(26^{\circ} \mathrm{C}\right)\end{array}$ & $\begin{array}{l}\text { Eau }(\mathrm{ml} / \mathrm{an} / \mathrm{j}) \\
\text { Eau/aliment }(\mathrm{ml} / \mathrm{g}) \\
\text { Eau/poids vif }(\text { p. } 100)\end{array}$ & $\begin{array}{c}376 a \\
2,36 a \\
20\end{array}$ & $\begin{array}{c}401 a \\
2,34 a \\
20\end{array}$ \\
\hline
\end{tabular}

Les moyennes figurant sur la même ligne et portant la même lettre ne sont pas significativement différentes $(p>0,05)$.

\section{Évaluation de l'intérêt économique de l'alimentation séparée}

L'un des objectifs de l'alimentation séparée, avec utilisation de céréales non concassées, est la réduction du coût de l'alimentation. Un calcul économique succinct est effectué pour évaluer le "prix d'intérêt" du complément protéique à utiliser : prix plafond en dessous duquel l'AS est plus économique que l'AC. La présente analyse prend en compte les paramètres économiques et zootechniques suivants :

$\mathrm{x}=$ prix d'intérêt du $\mathrm{kg}$ d'aliment complémentaire ;

$\mathrm{a}=$ prix du $\mathrm{kg}$ d'aliment complet ;

$\mathrm{b}=$ prix du $\mathrm{kg}$ de céréale ;

$c=$ part de la céréale dans la ration ingérée sur AS ;

$p=$ part du complément dans la ration ingérée sur $A S(p=1-c)$;

lac = indice de consommation sur $A C$;

las = indice de consommation sur AS.

A partir de ces paramètres, on peut effectuer les calculs économiques suivants:

- coût alimentaire/kg de gain sur $A C=a^{*} l a c$

- coût alimentaire/kg de gain sur $A S=\left(b^{*} c^{*} l a s\right)+\left(x^{*} p^{\star} l a s\right)$.
Le prix d'intérêt du complément est atteint lorsque les coûts d'alimentation $/ \mathrm{kg}$ de gain de poids sur AS et sur $A C$ s'équilibrent : $a^{*} l a c=b^{*} c^{*} l a s+x^{*} p^{\star} l a s ; d^{\prime}$ 'où :

$$
x=\frac{\left(a^{*} \mid a c\right)-\left(b^{*} c^{*} \mid a s\right)}{p^{\star} \text { las }}
$$

Cette formule est ensuite appliquée aux paramètres de la présente étude (essai 1) en tenant compte des prix des aliments relevés sur le marché pendant la période considérée : aliment complet (117 F CFA $/ \mathrm{kg}$ ) ; maïs (50 F CFA/kg) et mil (90 F CFA/kg). Pour ce calcul, les données zootechniques obtenues avec chaque céréale (maïs et mil) ont été regroupées. La procédure de calcul ainsi que les prix d'intérêt de l'aliment complémentaire sont indiqués dans le tableau IX pour chaque type de céréale.

\section{DISCUSSION}

Les performances obtenues avec les poulets de chair au cours de ces 2 expériences montrent que le mode d'alimentation séparée est apte à assurer un gain de poids adéquat des animaux, supérieur de 4 à 7 p.100 à la croissance obtenue avec un aliment complet présenté en farine, et comparable à celui permis par un aliment complet en granulé. Ces résultats sont en accord avec ceux signalés par COWAN et MICHIE (7) et MASTIKA et CUMMING (21). L'indice de consommation, l'efficacité de l'énergie et des protéines obtenues avec ces régimes expérimentaux montrent aussi que le poulet de chair valorise les nutriments issus d'un régime séparé avec une efficacité voisine de celle obtenue avec un aliment complet classique (32).

Sur la période totale (0-8 S), les régimes $C 1+$ maïs, $\mathrm{C} 1$ + mil et $\mathrm{C} 2$ + mil ont entraîné des consommations identiques. Les rations ingérées par les animaux étaient constituées pour $1 / 3$ de complément protéique et pour $2 / 3$ de céréales ; ce qui a abouti à une consommation moyenne de protéines de 16,5 à 17,5 g/animal/j comparable à l'ingestion observée sur aliment complet (16,3 g pour l'aliment farine et $17,5 \mathrm{~g}$ pour l'aliment granulé). Sur l'ensemble des périodes d'élovage, les animaux recevant les régimes séparés reconstituent par leur choix spontané une ration moyenne aux caractéristiques proches de celles des aliments complets. Avec l'âge, les poulets ont progressivement réduit la part d'aliment protéique dans leurs rations au profit des céréales: de 50 p. 100 au cours de la première semaine à $25-28$ p. 100 à la huitième semaine. Ce qui a entraîné une augmentation progressive du ratio énergie/protéines conformément aux recommandations nutritionnelles de l'INRA (17) bien qu'en saison sèche, on a observé des fluctuations importantes du ratio énergie/protéines entre 3 et 4 semaines d'âge (fig. 1). Ce résultat semble indiquer qu'en régime séparé, les poulets de chair "perçoivent" les différences de niveau nutritionnel des aliments offerts et ajustent leur ingéré protéique et/ou énergétique de façon à consommer une ration compatible avec leurs besoins de croissance (7). 
TABLEAU IX Évaluation de l'intérêt économique de l'alimentation séparée en fonction du prix de la céréale.

\begin{tabular}{|c|c|c|c|c|}
\hline & Maïs + complément & Mil + complément & $\mathrm{ACF}$ & ACG \\
\hline $\begin{array}{l}\text { Poids vif à } 8 \text { semaines }(\mathrm{g}) \\
\text { Gain de poids } 0-8 \text { semaines }(\mathrm{g}) \\
\text { Inaéré }\end{array}$ & $\begin{array}{l}2122 \\
2081\end{array}$ & $\begin{array}{l}2080 \\
2039\end{array}$ & $\begin{array}{r}2022 \\
1981\end{array}$ & $\begin{array}{l}2103 \\
2062\end{array}$ \\
\hline - céréale $(\mathrm{g})$ & 3332 & 3416 & - & - \\
\hline - complément $(\mathrm{g})$ & 1764 & 1624 & . - & - \\
\hline - Total $(\mathrm{g})$ & 5096 & 5040 & 4648 & 4872 \\
\hline - part de la céréale & 0,65 & 0,68 & . - & - \\
\hline - part du complément & 0,35 & 0,32 & - & - \\
\hline Indice de consommation & 2,45 & 2,47 & 2,35 & 2,36 \\
\hline Coût alimentaire $/ \mathrm{kg}$ de gain & $\left(50^{*} 0,65+x^{*} 0,35\right)^{\star} 2,45$ & $\left(90^{\star} 0,68+x^{\star} 0,32\right)^{\star} 2,47$ & 275 & 276 \\
\hline Prix plafond du complément (F CFA/kg) & 228 & 157 & - & - \\
\hline
\end{tabular}
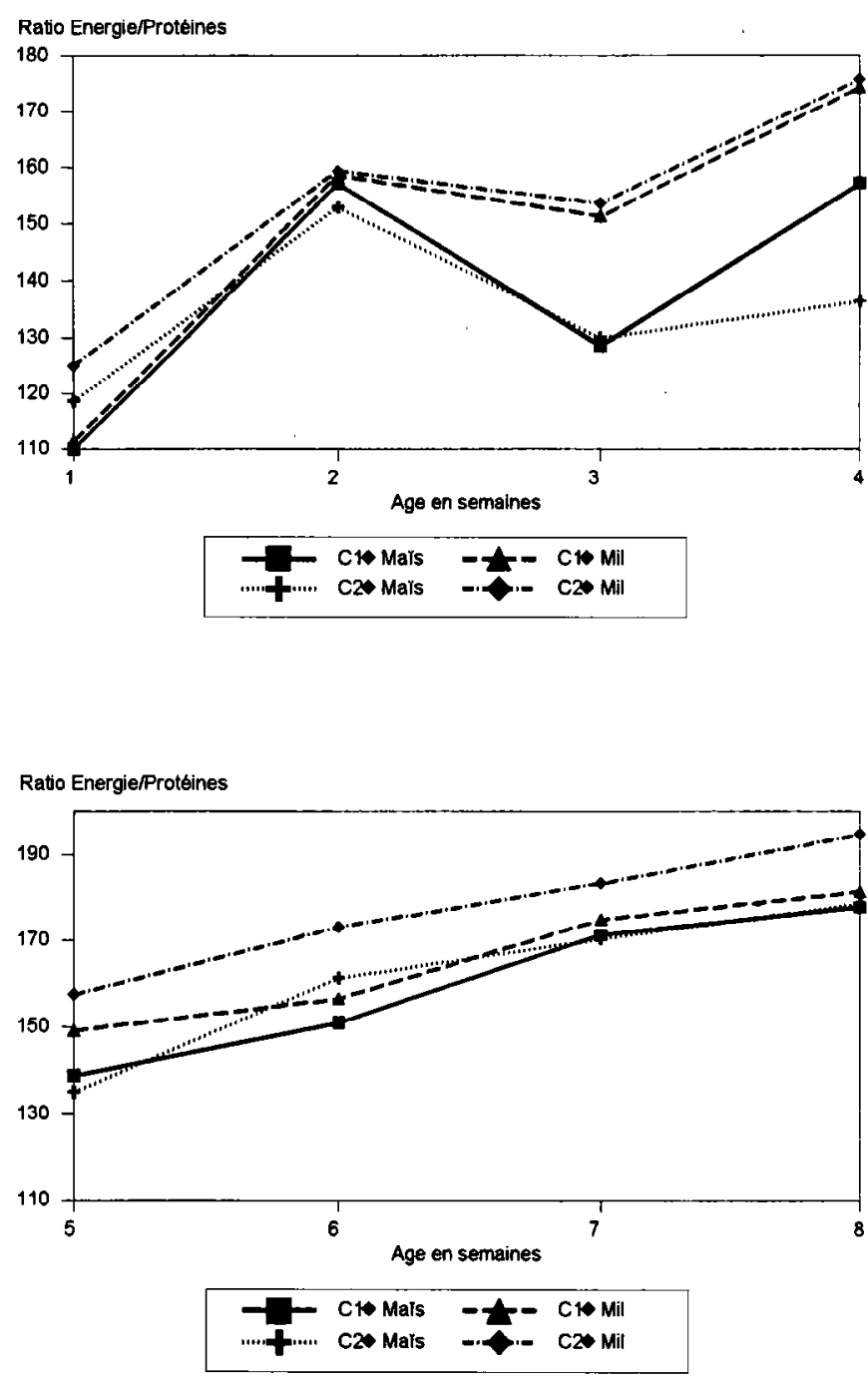

Figure 1 : Evolution du ratio énergie/protéines en périodes de démarrage (0-4S) et de croissance (5-8S).
Les résultats quelque peu irréguliers obtenus dans les mêmes conditions avec l'association C2 + maïs (sousconsommation du maïs) suggèrent que le besoin nutritionnel, bien que facteur majeur (27), ne constitue pas le seul déterminant du choix fait par l'animal. Plusieurs auteurs ont montré que le type de céréales $(8,9)$, la composition et la présentation des aliments (19) peuvent influer sur le choix de l'animal. Selon HUGHES (15), le choix fait par l'animal est, en définitive, le résultat d'un compromis entre les besoins nutritionnels de l'animal et l'appétibilité relative des aliments offerts au choix.

MASTIKA et CUMMING (21) ont par ailleurs indiqué que l'aptitude à opérer un choix correct requiert un apprentissage. Pendant cette période, l'animal établit le lien entre l'apparence physique et le niveau nutritionnel de l'aliment, d'une part et ses besoins physiologiques internes, d'autre part (3). La durée de l'apprentissage serait fonction du nombre d'aliments offerts au choix et de leurs différences de concentration en nutriments (27). Le taux élevé d'aliment protéique sélectionné en première semaine par des poulets de 28 jours mis brutalement en AS (expérience 2) confirme l'existence d'une période d'adaptation. Afin d'éviter un gaspillage de protéines chez les poulets de chair en AS, il semble plus indiqué de démarrer ce régime dès les premiers jours au moment où l'animal a besoin d'ingérer une ration à haute teneur en protéines. La comparaison des performances réalisées au cours des 2 expériences suggère un effet favorable de la saison fraîche sur l'ingestion d'aliment et le gain pondéral à 8 semaines (+ 25 p. 100). Cette différence n'apparaît qu'après 4 semaines d'âge et semble essentiellement due à la diminution importante d'ingéré alimentaire en milieu chaud (22). Ces résultats montrent l'effet de la saison sur le niveau d'ingestion et la croissance des poulets. Cependant, le dispositif expérimental utilisé n'a pas permis de mesurer l'interaction éventuelle entre la forme physique de l'aliment (granulé, farine) et la saison.

Les données obtenues sur la consommation hydrique montrent que dans l'environnement de l'étude, le poulet de chair boit 2 à 3 fois plus qu'il n'ingère d'aliment. Cette 
consommation est largement supérieure aux données indiquées pour les zones tempérées où le rapport eau/aliment est d'environ 1,5 (17). Ces résultats sont en accord avec le modèle proposé par SAUVEUR (28) qui estime que l'ingéré hydrique est multiplié par 2 entre 21 et $32^{\circ} \mathrm{C}$. La quantité d'eau bue et, surtout, le rapport eau/aliment semblent liés à la présentation de l'aliment. Les aliments présentés sous forme de grains (aliment séparé, aliment complet granulé) ont tendance à entraîner une consommation d'eau plus élevée que l'aliment présenté en farine. L'effet de la taille des particules sur la consommation d'eau est confirmé par.la comparaison maïs/mil. Les poulets consommant les gros grains de maïs boivent plus que ceux qui reçoivent les petits grains de mil. Ce comportement, qui a été également rapporté par STEVENSON (34), s'expliquerait probablement par le fait que l'eau bue sert également à ramollir les grains très secs et durs et à faciliter leur broyage dans le gésier. II semble s'atténuer lorsque le poulet devient plus âgé ; c'est-à-dire que la taille de son tube digestif augmente en fonction de la taille des particules alimentaires ingérées.

En définitive, l'AS apparaît comme une solution possible aux problèmes d'alimentation des poulets de chair en climat chaud. Grâce à l'utilisation directe de céréales non concassées, éventuellement produites sur l'exploitation, la technique d'AS pourrait permettre de réduire les achats d'aliments composés, les coüts de transport et de broyage ainsi que les problèmes liés aux difficultés de conservation. Toutefois, son intérêt dépendra des coûts du complément et du prix de vente de la céréale utilisée, par rapport au prix de l'aliment complet. L'analyse économique effectuée (tab. IX) montre, pour le maîs, que l'alimentation séparée est plus avantageuse tant que le coût du complément n'est pas supérieur au double du prix de l'aliment complet. Le mil est une céréale peu cultivée dans la zone d'étude (centre de la Côte d'Ivoire); ce qui explique son prix de vente élevé et son faible intérêt économique comme aliment de volailles dans cette région. En revanche, dans les régions du Nord, notamment au Sahel, où la culture du mil est répandue, son utilisation en alimentation séparée pourrait se révéler beaucoup plus intéressante. La mise au point d'aliments complémentaires à base de sous-produits locaux permettra une évaluation exacte de l'économie réalisée, au niveau d'une exploitation, avec cette méthode. Au niveau macroéconomique, la réduction des quantités d'aliments composés utilisés en aviculture pourrait se traduire par une réduction des dépenses d'investissement et d'exploitation des infrastructures de fabrication de provende.

\section{CONCLUSION}

Les résultats obtenus au cours de la présente étude montrent que la méthode d'alimentation séparée, à base de céréales non concassées et d'un complément protéique, constitue une alternative crédible à l'aliment complet classique. En plus de l'économie des coûts de transport et de broyage des céréales, l'utilisation de cette technique permettrait la valorisation directe des surplus de céréales et l'intégration d'un élevage avicole semiintensif, voire intensif, dans les systèmes agricoles locaux. Pour ce faire, il s'avère nécessaire de mettre au point un complément protéique d'excellente qualité mais de composition économiquement optimisée dans les conditions locales.

\section{REMERCIEMENTS}

Cette étude a bénéficié d'un financement du Fonds d'Aide à la Coopération (FAC) dans le cadre du programme "Recherchedéveloppement pour l'association agriculture-élevage en zone de savane". Les compléments protéiques utilisés ont été mis à notre disposition par l'UFAC (Vigny, France).

\section{BIBLIOGRAPHIE}

1.AIN BAZIZ (H.), GERAERT (P.A.), GUILLAUMIN (S.). Effects of high temperature and dietary composition on growth, body composition and energy retention in broilers. In: Proc. 8th Europ. Poult. Conf., Barcelona, 25-28 june 1990. p. 626-629.

2. BENABDELJELIL (K.), MERAT (P.). Test de types génétiques pour une production avicole locale au Maroc. Prod. anim., 1992, 5 (3) : 173-178.

3. BLUNDELL (J.E.), HILL (A.J.). Nutrition, serotonin and appetite: case study in the evolution of a scientific idea. Appetite, 1987, 8: 183-194.

4. BO'I'IJE (W.G.), HARRISON (P.C.). Etfect of carbonated water on growth performance of cockerels subjected to constant and cyclic heat stress temperatures. Poult. Sci., 1985, 64: 1285-1292.

5. BUSHMAN (D.). Alimentation en vue de la production d'oeufs en zones tropicales et subtropicales. Revue mond. Zootech., 1974, $12: 14-18$.

6. CARRE (B.), ROZO (E.). La prédiction de la valeur énergétique des matières premières destinées à l'aviculture. Prod. anim., 1990, 3 (3) : 163-169.

7. COWAN (P. J.), MICHIE (W.). Choice feeding of the turkey use of a high protein concentrate fed with either whole wheat barley oats or maize. Z. Tierphysiol. Tierernähr. Futtermittelkd, 1977, 39 (3): 124-130.

8. COWAN (P.J.), MICHIE (W.). Environmental temperature and choice feeding of the broiler. Br. J. Nutr., 1978, 40 (2): 311-315.

9. COWAN (P.J.), MICHIE (W.). Choice feeding of the male and female broiler. Br. Poult. Sci., 1978, 19 (2): 149-152.

10. DAGHIR (N.J.). Nutrient requirements of laying hens under high temperature conditivis. In: Poultry production in hot climates of iniddle east and fat east. 2nd international DLG-symposium,16-19 juin Goslar-Hahnenklee, RFA, 1985. p. 81-98.

11. DALE (N.M.), FULLER (H.L.). Effects of diet composition on feed intake and growth of chicks under heat stress. 1. Dietary fat levels. Poult. Sci., 1979, 58: 1529-1534.

12. DALE (N.M.), FULLER (H.L.). Effect of diet composition on feed intake and growth of chicks under heat stress. 2. Constant $v s$ cycling temperatures. Poult. Sci.,1980, 59: 1434-1441.

13. EL HUSSEINY (O.), CREGER (C.R.). The effect of ambiant temperature on carcass energy gain in chickens. Poult. Sci., 1980, 59: 2307-2311.

14. GERAERT (P.A.). Métabolisme énergétique du poulet de chair en climat chaud. Prod. anim.,1991, 4(3) : 257-267.

15. HUGHES (B. O.). The principles underlying choice feeding behaviour in fowls- with special reference to production experiments. Wld Poult. Sci. $J ., 1984,40(2): 141-150$. 
16. HURWITZ (S.), WEISELBERG (M.), EISNER (U.), BARTOV (I.), RIESENFELD (G.), SHARVIT (M.), NIV (A.), BORNSTEIN (S.). The energy requirements and performance of growing chickens and turkeys as affected by environmental temperature. Poult. Sci., 1980, 59: 2290-2299.

17. INRA. L'alimentation des animaux monogastriques : porc, lapin, volailles. Paris, INRA, 1980. $282 \mathrm{p}$.

18. JANSSEN (W.M.M.A.). European table of energy values for poultry feedstuffs. 2nd ed. Grafischbedrijf Ponson et Looijen bv Wageningen, 1988. p. $1-13$

19. KARUNAJEEWA (H.), THAM (S.H.). Choice feeding of the replacement pullet on whole grains and subsequent performance on laying diets. Br. Poult. Sci., 1984, 25 (1): 99-109.

20. MASTIKA (M.), CUMMING (R. B.). Performance of two strains of broiler chickens offered free choice from different ages. In: Energy and Environment in the Eighties. Proc. 4th Austral. Poult. and Stock Feed Convention, Perth, 12-15th October, 1981. p. 79-85.

21. MASTIKA (M.), CUMMING (R.R.). Effects of previous experience and environmental variations on the performance and pattern of feed intake of choice fed and complete fed broiler. Microbiology and nutrition. University of New England. In: FARREL (J) ed. Recent advances in animal nutrition in Australia. 1981. p. 260-282.

22. OSMAN (A.M.A.). Einfluss von Stalltemperatur und Geschlecht in Abhängigkcit vom Mastalter auf die Mastleistung, den Schlachtkörperwert und die Fleischbeschaffenheit beim Masthuhn. Th. Doct., Université de Kassel, RFA, 1988. 198 p.

23. PICARD (M.). Heat effects on the laying hen: Protein nutrition and food intake. In: Proc. 5th Europ. Symp. Poult. Nut. Maale Hachamisha, Israel, 27-31 Oct. 1985. p. 65-72.

24. PICARD (M.), PLOUZEAU (M.). Nutritional aspects of adaptation of poultry to heat stress: laying hens. In: Proc. 7th Europ. Symp. Poult. Nutr., I loret de Mar Giro, Espagne, June 19-21, 1989. p. 83-98.

YO (T.), PICARD (M.), GUERIN (H.), DAUVILLIERS (P.). Free choice feeding (whole cereal grains + pelleted complementary feed) of broilers under hot climate. Revue Élev. Méd. vét. Pays trop., 1994, 47 (3) : $319-327$

Food intake and growth of broilers are limited by high environmental temperatures. Free choice feeding (FCF), including whole grains (cereals) and a complementary concentrate, was compared to a complete feed, ground (CFG) or pelleted (CFP) to study the ability of broilers to adjust their energy and protein intake to their needs. 1012 "JV15" broilers were divided into 24 groups in two experiments, either during dry season (DS) or cooler rainy season (RS). Each group received one of six diets: CFG, CFP or one of four FCF combinations composed of maize or millet and of two complementary concentrates. 56 day old broilers weighed $25 \%$ more during RS than in DS. Whatever the season, FCF fed broilers weighed $4.7 \%$ more than the CFG fed broilers. On the contrary, the liveweight of FCF and CFP fed broilers were similar during the DS (CFP not tested during RS). A week of adaptation to FCF was necessary ; after this delay, energy/protein ratio was similar for the 4 FCF diets and CFP. With this technic, the direct use of grain is possible without transport, grinding, mixing and pelleting. The shadow price of complementary feed depends on those of grains and CFP.

Key words : Broiler - Feeding system - Food ressource - Growth - Tropical climate - Côte d'Ivoire.
25. PICARD (M), SAUVEUR (B.). FENARDJI (F), ANGULO (I), MONGIN (P.). Ajustements technico-économiques possibles de l'alimentation des volailles dans les pays chauds. Prod. anim., 1993, 6 (2) : 87-103.

26. ROBINSON (D.). Performance of laying hens as affected by split time and split composition dietary regimens using ground and unground cereals. Br: Poult. Sci, 1985. 26 (3): 299-309.

27. ROSE (S.P.), KYRIAZAKIS (I.). Diet selection of pigs and poultry. Proc. Nutr. Soc., 1991, 50: 87-98.

28. SAUVEUR (B.). Reproduction des volailles et production d'oeufs. Paris, INRA, $1988.449 \mathrm{p}$.

29. SAXENA (H.C.). Rearing broilers in sub-tropics: cool is comfortable. Wld Poult., 1990, 6 (4): 21.

30. SCHOLTYSSEK (S.). Beitrag zur Wahlfuetterung von Broilern. Arch. Gefluegelk., 1982, 46 (6): 243-247.

31. SEEMANN (G.). Mastleistung und Schlachtkoerperqualitaet nach Wahlfuetterung von Broilern. 2. Arch. Gefluegelk., 1984, 48 (3): 100-106.

32. SINURAT (A. P.), BALNAVE (D.). Free-choice feeding of broilers at high temperatures. Br. Poult. Sci.. 1986, 27 (4): 577-584.

33. SMITH (A.J.). Poultry. London. The Mcmillan Press ltd. 1990. 218 p.

34. STEVENSON (M.H.). The nutritional value of cassava root meal in laying diets. J. Sci. Fd. Agric. 1984. 35: 36-40.

35. VALENCIA (M.E.), MAIORINO (P.M.), REII) (B.I.). Fnergy utilization by laying hens. 2. Energetic efficiency and added tallow at 18.3 and $35^{\circ}$ C. Poult. Sci., 1980, 59: 2071-2076.

YO (T), PICARD (M), GUERIN (H), DAUVILLIERS (P) Alimentación libre (granos enteros de cereales + pienso complementario en gránulos) para pollos de engorde en clima cálido. Reviue Élev. Méd. vét. Pays trop.. 1994, 47 (3) : 319-327

Las temperaturas elevadas limitan el consumo alimenticio y el crecimiento de los pollos de engorde. Se comparó una alimentación libre $(\mathrm{AL})$, incluyendo granos enteros de cereales y un pienso complementario, con un pienso completo de harina (PCH) o de gránulos (PCG) con objeto de estudiar la aptitud de los pollos para acomodar la ingestión de energía y de proteínas a las necesidades nutritivas. Durante dos ensayos realizados en la estación seca (ES) y la estación lluviosa (EL), más fresca, se observaron 1012 pollos JV15 repartidos entre 24 grupos ; cada uno de dichos grupos recibió 6 regimenes alimenticios : PCH, PCG y 4 combinaciones de $\mathrm{AL}$ comprendiendo maiz o mijo con dos fórmulas de piensos complementarios. Los pesos a 56 días de edad fueron más elevados con EL que con ES ( 25 p. 100). Cualquiera que sea la estación, los crecimientos con AL fueron de 4 a 7 p. 100 superiores a los obtenidos con PCH. En cambio, en ES los rendimientos de los grupos con AL y PCG estaban globalmente idénticos (PCG no experimentado en EL). La AL necesitó una semana de adaptación, después de lo cual la relación energía/proteínas fue próxima de la de los piensos completos. Permite utilizar las cereales locales sin gastos de transporte, de molienda, de mezcla o de granulación. Se puede determinar el precio tope del pienso complementario mediante los de las cereales y del PCG.

Palabras clave : Pollo de engorde - Sistema de alimentación - Recurso alimentario - Crecimiento - Clima tropical - Côte d'Ivoire. 